\title{
Inductive powering of subcutaneous stimulators: key parameters and their impact on the design methodology
}

\author{
Carmen Godfraind (1,2), Adrien Debelle (2), Laurent Lonys (2), Vicente Acuña (2), Pascal \\ Doguet (1), Antoine Nonclercq (2) \\ (1) Synergia Medical, Mont-Saint-Guibert, Belgium; (2) Université Libre de Bruxelles, \\ Belgium \\ This article is distributed under the terms of the Creative Commons Attribution Noncommercial License (CC BY-NC 4.0) which \\ permits any noncommercial use, distribution, and reproduction in any medium, provided the original author(s) and source are credited.
}

\begin{abstract}
Inductive powering of implantable medical devices involves numerous factors acting on the system efficiency and safety in adversarial ways. This paper lightens up their role and identifies a procedure enabling the system design. The latter enables the problem to be decoupled into four principal steps: the frequency choice, the magnetic link optimization, the secondary circuit and then finally the primary circuit designs. The methodology has been tested for the powering system of a device requirering a power of $300 \mathrm{~mW}$ and implanted at a distance of 15 to $30 \mathrm{~mm}$ from the outside power source. It allowed the identification of the most critical parameters. A satisfying efficiency of $34 \%$ was reached at $21 \mathrm{~mm}$ and tend to validate the proposed design procedure.
\end{abstract}

Key Words: implanted neurostimulators, inductive powering, design methodology, efficiency optimisation

Eur J Transl Myol 201626 (2) 155-160

The use of inductive powering systems for implantable stimulators has risen unceasingly over the last years since this non-invasive technology offers an unlimited controllable power source, coping with problems of nonrechargeable batteries or percutaneous electrodes leads. Batteries present a limited lifetime requiring regular surgical operations for replacement. Moreover, the limitation on the size of the implanted systems strongly restricts the energy budget of the battery. ${ }^{1}$ Inductive powering is based on the magnetic coupling of two coils: one (coil 1) outside the body, transmitting the energy, and another (coil 2) implanted within the body, receiving the energy. This energy is delivered to a rechargeable battery or can be used directly by the implant to perform the stimulation. The energy provided to the primary circuit by a dc supply source is converted into an alternative signal by a power amplifier (PA). This signal flows through the primary coil and generates an oscillating magnetic field sensed by the secondary coil inside the body. Magnetic energy is then transformed to electrical energy via a resonant secondary circuit. Finally, it is turned into a dc signal by a power rectifier. Despite its widespread use, inductive powering technology still presents numerous challenges when it comes to applying it to implantable medical devices. The complexity of the interactions between the different factors on which the power transfer system (PTS) relies implies an in-depth study leading to the identification of a reliable and efficient design procedure The proposed paper consolidates current literature to produce a coherent methodology that is validated on a case study through simulations and measurements.

\section{Materials and Methods}

The global design methodology chart flow is presented in Fig. 1. The system can be decoupled according to its physical and electrical parts. Two main aspects are thus to be considered for the design methodology: the magnetic link design and the electronic design. The magnetic link design is based on the optimization of the magnetic coupling between the transmitter and the receiver, and aims to minimize the power loss between them. It only depends on the material properties and the coil geometries and placement. It is therefore the first step of the design procedure. The electronic design can in itself be split into two steps: the design of the secondary circuit and then the determination of the primary circuit components. This division into subsystems is convenient to tune each oscillating circuit individually. It reduces the risk of having to change the secondary coil or circuit after a modification of the primary circuit. Subsequently, it limits the number of 


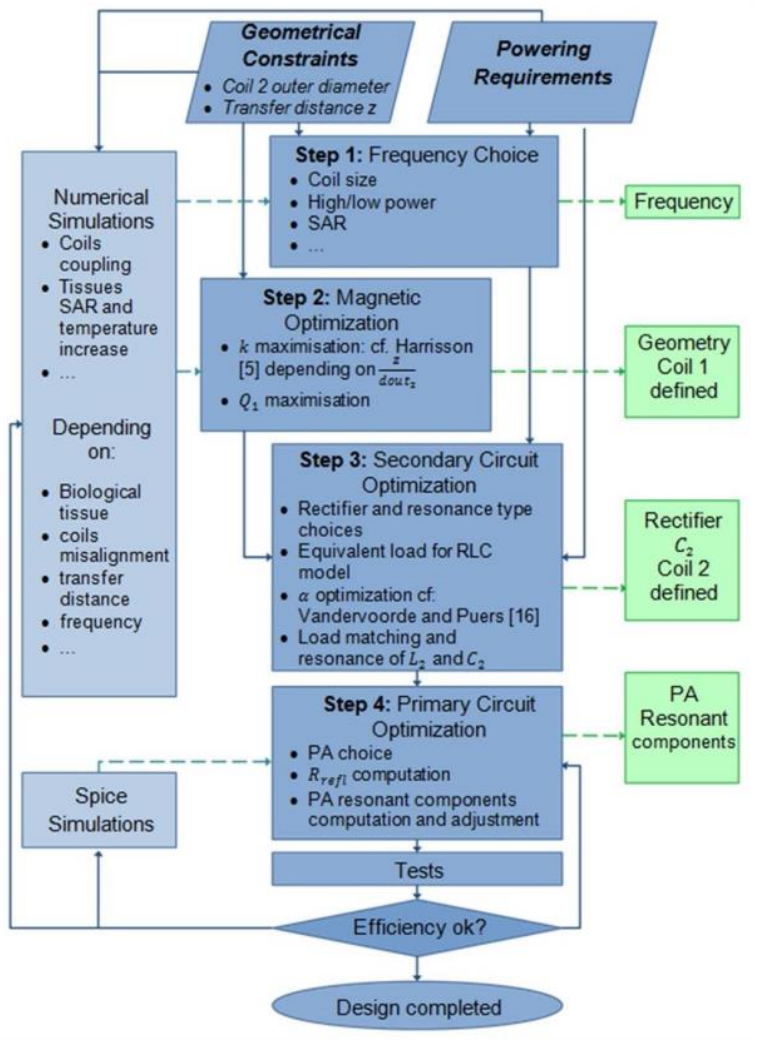

Fig 1. Design methodology chart flow presenting the different steps to follow from the initial requirements and constraints to the obtainment of an efficient power transfer system, and the possible simulations to perform in parallel in order to improve the analytical and experimental process.

iterations of the design procedure. In this paper, we present an example of an application for this methodology with given specifications: constant $60 \mathrm{~mA}$ current through a load at $5 \mathrm{~V}$ nominal voltage; a nominal transfer distance $Z$ of $15 \mathrm{~mm}$ between transmitter and receiver coils, while the outer diameter $d_{\text {out }}$ of the implanted coil should not exceed $26 \mathrm{~mm}$.

\section{Step 1: Frequency Choice}

The choice of the operating frequency is highly critical. The magnetic link efficiency increases when the coupling coefficient $k$ and the quality factors of the coils $Q_{1}$ and $Q_{2}$ increase (see section $B$.). Regarding the coupling coefficient, it can be enhanced by using larger coils that imply using a lower frequency. Besides, a decrease in frequency leads to the reduction of the quality factors of the coils. On the other hand, the choice of a higher frequency leads to a higher energy absorption by the patient's tissues. Moreover, higher frequencies will affect the power amplifier and rectifier efficiencies and enlarge the undesired equivalent series resistance $E S R$ of the coils because of the skin and proximity effects. $^{2}$ Besides, designers should also keep in mind the allowable frequency allocation for medical devices 3

Considering those facts, inductive powering systems for medical implants generally operate in the range of hundreds of kilohertz to several tens of Megahertz. ${ }^{4-}$ ${ }^{12}$ To clarify the problem, the choice of the working frequency has been defined at the beginning of the procedure. Considering the small size of the coils generally designed for industrial purposes, $6.78 \mathrm{MHz}$, the lowest FCC-approved ISM frequency ${ }^{13}$ was chosen

\section{Step 2: Magnetic Link Design}

The magnetic link efficiency between the transmitter and receiver coils is directly proportional to the design factor $X=k^{2} Q_{1} Q_{2}{ }^{1,4}$, where $k$ is the coupling coefficient, a dimensionless quantity equal to the fraction of the magnetic flux generated by the primary coil that flows through the secondary coil, and where $Q_{i}$ are the quality factors of the respective coils characterizing the ratio of the stored energy to the energy dissipated in the coils. Eq. 1 is an approximation of the Q-factors valid for inductive powering coils ${ }^{4,5}$ :

$$
Q_{\mathrm{i}}=\frac{\omega L_{\mathrm{i}}}{R_{\mathrm{i}}}
$$

where $\omega$ is the angular resonant frequency, $L_{i}$ and $R_{i}$ are respectively the self-inductances and the ESR of the coils $i$.

Optimizing the magnetic link is performed by maximizing the coupling coefficient and the coils quality factors. However, there is a limit on $Q_{1}$ since it could lead to a high and dangerous voltage at the extremities of the coil $1{ }^{4}$ The coupling coefficient does not depend on the circuit topology. Also, since the mutual inductance $M$ is proportional to $N_{1} N_{2}$, and $L_{i}$ proportional to $N_{i}^{2}, k$ does not depend on the number of coil turns. The main focus of the magnetic design consists thus in maximizing $k$ by fixing appropriate coil geometries as suggested by R. R. Harrisson. ${ }^{4}$ The latter uses the current sheet approximation to analytically compute the inductance of planar spiral coils. ${ }^{4,14}$ This type of coils is the most appropriate for subcutaneous applications as they offer the most comfort $^{6}$ and as they present a remarkable mutual inductance. ${ }^{7,8} M$ can be estimated by calculating the mutual inductances between two pairs of circular single coils equivalent to the spiral coils. ${ }^{4,7}$ Harrisson's methodology for maximum coupling leads to the coils outer and inner diameters $d_{\text {out }_{i}}, d_{\text {in }_{i}}$ depending on the ratio of the transfer distance and the outer diameter of the receiver $\frac{z}{\text { dout }_{2}}$, which are the system main geometrical constraints. Once this geometry is fixed, coil 1 can be defined, enhancing $Q_{1}$ by determining the appropriate number of turns and choosing wires of specific diameters. Proximity and skin effects should also be considered: Litz wires are recommended to reduce the coils $E S R{ }^{5,16,17}$ Only the gross geometry of 


\section{Inductive powering of subcutaneous stimulators}

Eur J Transl Myol 26 (2) 155-160

Table1. Magnetic Design Outputs (where $z$ is the transfer distance between the coils)

\begin{tabular}{|l|c|c|}
\hline & Coil 1 & Coil $\mathbf{2}$ \\
\hline Outer diameter $(\mathbf{m m})$ & 56.0 & 25.4 \\
\hline Inner diameter $(\mathbf{m m})$ & 11.2 & 16.3 \\
\hline Turns number & 26 & Not defined \\
\hline Coupling coefficient & \multicolumn{2}{|c|}{$0.15($ at z=15mm) } \\
\hline
\end{tabular}

the coil 2 is defined at this stage: $L_{2}$ and $Q_{2}$ impact the secondary circuit efficiency and will be designed to match the load corresponding to the power requirements. The use of magnetic materials could improve the coupling between the coils, but this has not been considered in the present paper. Moreover, magnetic materials can have a dangerous impact when it comes to MRI compatibility.

Table 1 presents the results obtained at the end of the magnetic design for our example. Coil 1 is defined and has an inductance of $19 \mu \mathrm{H}$.

\section{Step 3: Secondary Circuit Design}

The first part of the secondary circuit design consists in choosing a rectifier. Here, we suggest a reliable and simple full-bridge rectifier ${ }^{9}$ since the secondary voltage is expected large enough to provide the required output voltage despite the two diodes voltage drops. An equivalent load $R_{\text {load }}$ representing the rectifier and the output load $R_{D C}$ is calculated according to Eq. $2 .{ }^{12}$ The circuit is then represented by a simple RLC model.

$$
R_{\text {load }}=\frac{R_{D C}}{2}\left(1+\frac{2 V_{\text {diode }}}{V_{\text {out }}}\right)
$$

where $V_{\text {out }}$ the output voltage of the PTS and $V_{\text {diode }}$ the diodes forward voltage. Secondly, the choice of the resonant capacitor $C_{2}$ and the receiver coil configuration is made. Series-resonant drivers act as current sources while parallel-resonant drivers act as voltage sources. The decision depends on the value of the load and on the rectifier. A parallel circuit appeared the most suited for this system. $1,11,12$

Then, the number of turns $N_{2}$ and the inductance $L_{2}$ of the coil 2 is determined. In order to enhance the system efficiency, it is important to match the resonant components $\mathrm{L}_{2}$ and $\mathrm{C}_{2}$ to the load. It is done via the optimization of the efficiency with respect to the parameter $\alpha$ defined by G. Vandervoorde and R. Puers ${ }^{11}$ as a dimensionless quantity corresponding to the ratio of $\mathrm{R}_{\text {load }}$ to the reactance of $\mathrm{C}_{2}$. Depending on the resonant circuit type, the efficiency formula varies and is developed in. ${ }^{1}$ The $\alpha$ obtained at maximum efficiency $\alpha_{\text {opt }}$ (Eq. 4) ${ }^{11}$ allows the computation of $\mathrm{C}_{2}$ in function of the load (Eq. 5). $\mathrm{L}_{2}$ is finally deduced from equation of parallel RLC resonance (Eq. 6).

$$
\begin{gathered}
\alpha=\omega C_{2} R_{\text {load }} \\
\alpha_{\text {opt }}=\frac{Q_{2}}{\sqrt{1+X}}=\alpha @ \frac{\partial \eta}{\partial \alpha}=0 \\
C_{2}=\frac{\alpha_{\text {opt }}}{\omega R_{\text {load }}}
\end{gathered}
$$

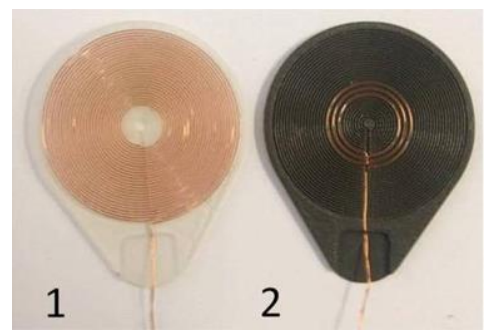

Fig 2. Designed Coils where the coil 1 and 2 are respectively the primary and the secondary coils.

$$
\omega=\sqrt{\frac{1}{L_{2} C_{2}}-\frac{1}{R_{\text {load }}^{2} C_{2}^{2}}}
$$

Since the calculation of coil 2 parameters depends on its quality factor $Q_{2}$, the previous computations consist in several iterations, starting with a first estimation of $Q_{2}$ (typically between 10 and 200). ${ }^{16}$ At each iteration, new values are calculated for $C_{2}, L_{2}$ and $E S R_{2}$ until the value of $Q_{2}$ is stable. Once $L_{2}$ is determined, and since the geometrical dimensions have been defined during the magnetic design, $N_{2}$ is given by to the current sheet approximation. ${ }^{14}$ The circuit on the right of Fig 3 is obtained at this stage. Coil 2 is now defined: it has 3 turns (see Fig. 2) and an inductance of $0.48 \mu \mathrm{H}$. The impedance of the secondary circuit will be seen by the primary circuit through the magnetic link: it is called the reflected impedance $R_{\text {refl }}$ which is real if the operating frequency is equal to the secondary resonant frequency. Depending on the coupling strength, $R_{\text {refl }}$ will influence more or less the transmitter circuit. ${ }^{18-20}$ This is the reason why the secondary circuit is designed before the primary.

\section{Step 4: Primary Circuit Design}

Once the secondary circuit has been fixed, the primary circuit is conceived. First of all, the PA is chosen. It is then necessary to adapt the driving circuit components in function of the coil impedance, the frequency, the reflected impedance (if the coupling is important enough to have a significant influence on the PA operation) and the coils coupling. The class E PA is chosen because of its high efficiency especially for frequencies in the range of $3 \mathrm{MHz}$ to $1 \mathrm{GHz} .{ }^{19}$ The PA efficiency is essentially due to the minimization of the power loss obtained by preventing the current to flow through the transistor when its drain-source voltage $V_{d s}$ is not zero, and vice-versa. It is necessary to adapt the components to the primary load i.e. to $R_{\text {refl }}$ (Eq. 7 for a parallel secondary circuit) $E S R_{1}$, and the driver output impedance in order to approach the targeted signals.

$$
R_{\text {refl }}={\frac{(\omega M)^{2}}{E S R_{2}+R_{\text {load }}}}^{18}=k^{2} L_{1} \omega{\frac{\alpha Q_{2}}{\alpha+Q_{2}}}^{1}
$$




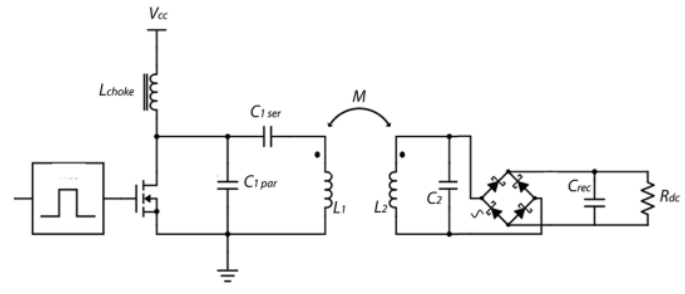

Fig 3. Power transfer system PTS obtained at the end of the design methodology for our example.

Frederick H. Raab ${ }^{12,21}$ designed equations particularly appropriate for inductive powering systems allowing to get the tuning components estimation ${ }^{1}$. The effective components values differ from those ideally designed: it is possible to adjust them to obtain effectively a high efficiency operation by using Sokal's method. ${ }^{19}$ Once this adjustment is done, the complete design optimizing the efficiencies of the magnetic link, the secondary and the primary circuits, is finished. The circuit (Fig. 3) can be tested for its output power and efficiency.

\section{Results}

The results of this section correspond to coils laterally and angularly aligned separated by an air gap of 10 to $30 \mathrm{~mm}$. Fig. 4 represents the total efficiency (i.e. the ratio of the output power at $R_{D C}$ to the input power from the supply), the efficiency before rectification (i.e. the ratio of the power received at the secondary coil to the input power from the supply) and the output power of the PTS. This result is obtained by varying the value of the $\mathrm{C}_{1_{\text {ser }}}$ capacitor (see Fig. 3) around the ideal class $\mathrm{E}$ operating point for a switch command frequency of $6.78 \mathrm{MHz}$. The maximum total efficiency does not correspond to the ideal class E operating point (measure 6 on Fig. 1) where the drain voltage and its derivative are zero at transistor switch on. However, that point corresponds to the maximal efficiency before the signal rectification. At this operating point, the overall efficiency is $17.8 \%$ and the efficiency without the rectification is $49.3 \%$. Since the high rectification losses reduce consequently the overall efficiency, Schottky diodes are used for the rectifier in the subsequent text (e.g. Fig. $5 \& 6$ ). Regarding the output power, it rises until its maximum of $325 \mathrm{~mW}$ corresponding to a system efficiency of $24.4 \%$. Fig. 4 helps to understand the implication of the different sources of power loss: the $\mathrm{PA}$, the loose coupling, and the rectifier. Since the magnetic loss is related to the link gain depending only on the geometry, the principal factor influencing the efficiency before rectification is the PA. This explains that the best efficiency before rectification is found at the class E operating point. For subsequent measures (for which the switch command frequency is higher than the resonant frequency), the decrease of output power and efficiency is due to the PA losses. When the PA is switched at a frequency lower than the frequency needed

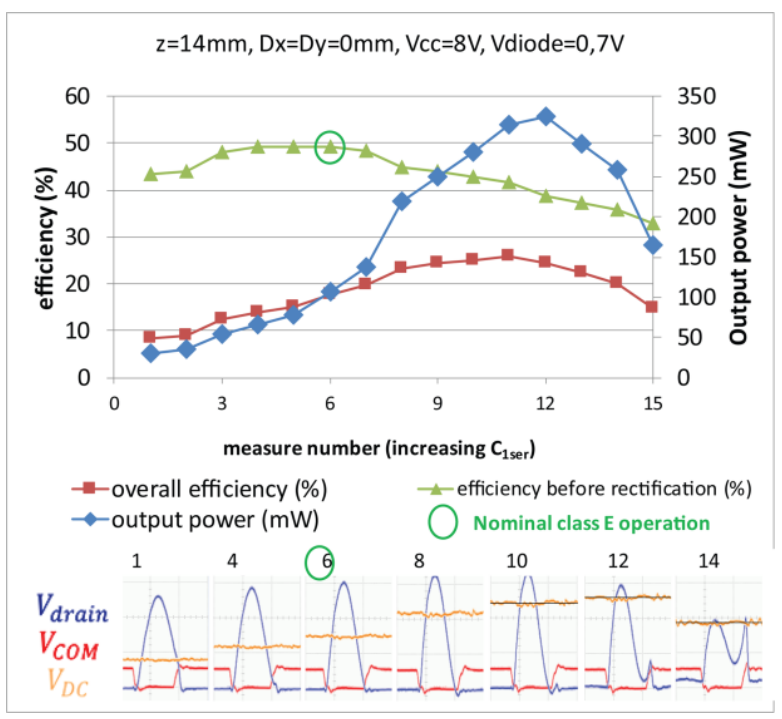

Fig 4. Efficiency before and after rectification, output power and screenshots of drain voltage $V_{\text {drain }}$, gate voltage $V_{\text {COM }}$ and voltage at the load $V_{D C}$ for different class $E$ power amplifier operating points.

to get the right class E operation, the output power and the overall efficiency rise despite the class E losses: for those operating frequencies, the input consumption increases which also increases the primary coil voltage. Since the gain is nearly constant, the secondary voltage increases too and enhances the rectifier efficiency. ${ }^{9}$ The decrease of the output power and of the efficiency only appears when these power gains cannot compensate the losses in the PA switch anymore. Fig. 5 shows that the maximal transfer efficiency and output power are obtained around a transfer distance of $16 \mathrm{~mm}$. For $8 \mathrm{~V}$ supply, the efficiency reaches $38.1 \%$ and the output power equals $244 \mathrm{~mW}$. The improvement in efficiency compared to Fig. 4 (where the maximum efficiency was only $24.4 \%$ ) is due to the use of Schotkky diodes. The transfer distances considered for the subcutaneous implant are generally ranging from 15 to $20 \mathrm{~mm}$. At $20 \mathrm{~mm}$, the efficiency is still of $32.6 \%$ for an output power of $208 \mathrm{~mW}$. Fig. 6 shows that the PTS enables to deliver more than the targeted $300 \mathrm{~mW}$ required by the initial power demand to the load for a supply voltage of $9 \mathrm{~V}$ and a transfer distance of $21 \mathrm{~mm}$. The corresponding efficiency is of $33.3 \%$.

\section{Discussion}

Tests on efficiency and output power tend to validate the proposed design procedure: it leads to a system presenting a large efficiency (e.g. up to $34 \%$ at $21 \mathrm{~mm}$ ). For the secondary circuit, the rectification efficiency can become highly critical, in particular for low power applications with weak link gain. Therefore, high 


\section{Inductive powering of subcutaneous stimulators}

Eur J Transl Myol 26 (2) 155-160

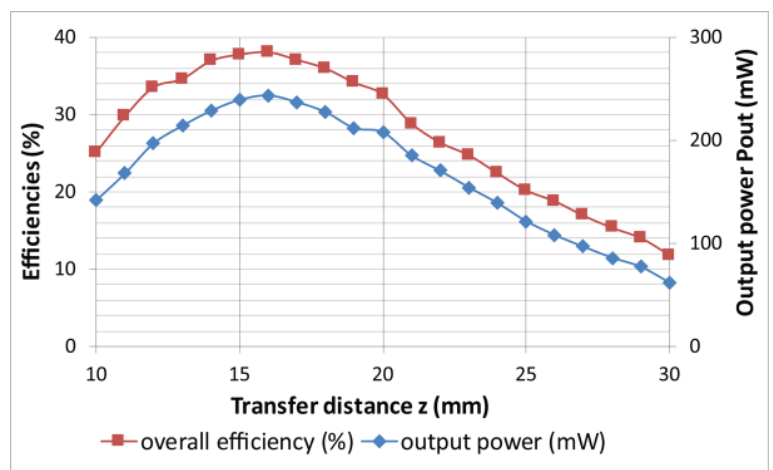

Fig 5. System efficiency and output power variation with the transfer distance $z$. Supply voltage $V_{c c}=8 V$; Rectifiction diodes forward voltage $V_{\text {diode }}=0.35 \mathrm{~V}$.

primary voltage is usually needed and class E PA are recommended. A trade-off can be found between the losses of the primary and the secondary circuits in order to maximize the efficiency and the expected output power over the required range of transfer distance.

This methodology presents some limitations: depending on the initial requirements, some components can show very small values, comparable to parasitic impedances, therefore having fluctuating and unexpected impacts on the results. This justifies the necessity of an iterative testing in order to guaranty the optimal power transfer. Moreover, the class E PA is very sensitive to frequency shifts. Coils misalignment or deformations therefore become quickly a problem. Further improvements can be brought by adding a feedback regulation on the operating frequency of the class E PA. Moreover, numerical simulations (see Fig. 1) allow considering coils misalignments, complex geometries, and heating of tissues. The development of numerical models will thus induce further improvement of the design procedure that, hopefully, will enable researchers to identify more easily the critical features of their design and to proceed in a consistent way. We hope that this methodology can be applied to any type of implantable inductively powered system. Further work will include the validation of this methodology on various hardware systems comprising classical telemetry based on the inductive link and more particularly $\mathrm{LSK}^{10}$, benefiting from the coils coupling optimisation.

\section{Contributions}

CG performed a deep review of the existing litterature. She also designed, tuned and tested the electronics circuits and performed a practical implementation of the proposed methodology. AD and VA helped during the elaboration of the methodology and the prototype testing. LL gave his expertise on the power requirements of neurostimulators and reviewed the work. PD gave his expertise on the wireless power transfer for implantable

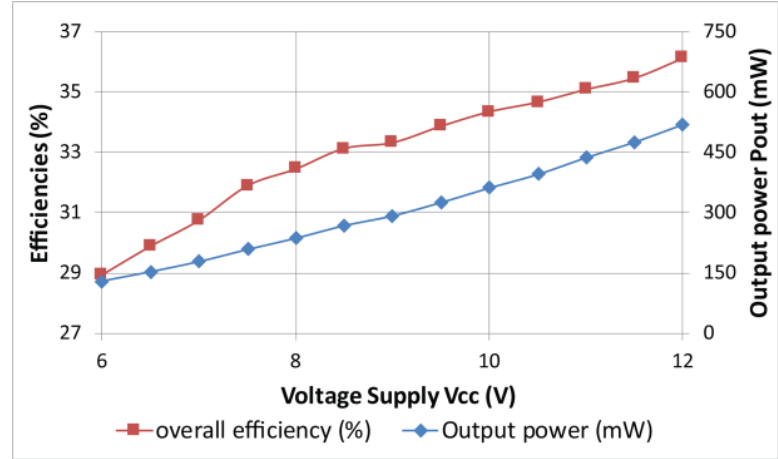

Fig 6. System efficiency and the output power variation with the supply voltage. Power transfer distance $z=21 \mathrm{~mm} ;$ Rectification diodes forward voltage $V_{\text {diode }}=0.35 \mathrm{~V}$.

devices and helped designing the driving circuit of the class E PA. This work was supervised by PD and AN.

\section{Acknowledgements}

Research supported by Synergia Medical, Mont-SaintGuibert, Belgium.

\section{Conflict of Interest}

The authors declare no potential conflict of interests.

\section{Corresponding Author}

Carmen Godfraind, Syngeria Medical, Mont-SaintGuibert, Belgium

E-mail: carmen.godfraind@ synergiam.com

E-mails of coAuthors

Adrien Debelle: addebell@ulb.ac.be

Laurent Lonys: 1lonys@ulb.ac.be

Vicente Acuña: Vicente.Acuna.Otarola@ulb.ac.be Pascal Doguet: pascal.doguet@synergiam.com Antoine Nonclercq: anoncler@ulb.ac.be

\section{References}

1. Schuylenbergh $\mathrm{K}$ Van, Puers $\mathrm{R}$. Inductive Powering: Basic Theory and Application to Biomedical Systems. Analog Circuits And Signal Processing 2009.

2. Bosshard R, Muhlethaler J, Kolar JW, Stevanovic I. Optimized magnetic design for inductive power transfer coils. Conf Proc - IEEE Appl Power Electron Conf Expo - APEC 2013:1812-1819.

3. ECC within CEPT. The European Table of Frequency Allocations and Applications in Frequency Range $8.3 \mathrm{kHz}$ to $3000 \mathrm{GHz}$; 2013.

4. Harrison RR. Designing Efficient Inductive Power Links for Implantable Devices. 2007 IEEE Int Symp Circuits Syst 2007:2080-2083.

5. Artan NS, Patel RC, Ning C, Chao HJ. Highefficiency wireless power delivery for medical implants using hybrid coils. Conf Proc IEEE Eng Med Biol Soc 2012:1683-1686. 


\section{Inductive powering of subcutaneous stimulators}

Eur J Transl Myol 26 (2) 155-160

6. Ma Q, Haider MR, Yuan S, Islam SK. Poweroscillator based high efficiency inductive powerlink for transcutaneous power transmission. Midwest Symp Circuits Syst 2010:537-540.

7. Mutashar S, Hannan M a., Samad S a., Hussain A. Analysis and optimization of spiral circular inductive coupling link for bio-implanted applications on air and within human tissue. Sensors (Switzerland) 2014;14:11522-11541.

8. Chaoui M, Perdriau R, Ghariani H, Lahiani M. Design of a class-E transcutaneous energy transmitter for an implantable system. Microelectron Int 2012;29:22-34.

9. Lenaerts B, Puers R. Power Converters and Voltage Regulators. In: Power. Vol ; 2009.

10. Tang Z, Smith B, Schild JH, Peckham PH. Data transmission from an implantable biotelemeter by load-shift keying using circuit configuration modulator. IEEE Trans Biomed Eng 1995;42:524528.

11. Vandevoorde G, Puers R. Wireless energy transfer for stand-alone systems: A comparison between low and high power applicability. Sensors Actuators, A Phys 2001;92:305-311.

12. Lenaerts B, Puers R. Omnidirectional Inductive Powering for Biomedical Implants. Analog Circuits and Signal Processing. Springer; 2009.

13. ITU. Rec. ITU-R SM.1056: Limitation of radiation from industrial, scientific and medical (ISM) equipment 1994.

14. Mohan SS, Hershenson MDM, Boyd SP, Lee TH. Simple accurate expressions for planar spiral inductances. IEEE J Solid-State Circuits 1999;34:1419-1420.

15. Pospisilik M, Kouril L, Motyl IVO, Adamek M. Single and Double Layer Spiral Planar Inductors Optimisation with the Aid of Self-Organising Migrating Algorithm In Proceedings of the 11th WSEAS International Conference on Signal Processing, Computational Geometry and Artificial Vision. Venice: WSEAS Press (IT) 2011 Aug 23

16. Sun T, Xie X, Wang Z. Wireless Power Transfer for Medical Microsystems. Springer; 2013.

17. Schuder JC, Gold JH, Stephenson HE. An inductively coupled RF system for the transmission of $1 \mathrm{~kW}$ of power through the skin. IEEE Trans Biomed Eng 1971;18:265-273.

18. Ko WH, Liang SP, Fung CDF. Design of radiofrequency powered coils for implant instruments. Med Biol Eng Comput 1977;15:634-640.

19. Sokal N. Class-E high-efficiency RF/microwave power amplifiers: Principles of operation, design procedures, and experimental verification. Analog Circuit Des 2002:269-301.

20. Yang T, Zhao C, Chen D. Feedback analysis of transcutaneous energy transmission with a variable load parameter. ETRI J 2010;32:548554.

21. Raab F. Idealized operation of the class $E$ tuned power amplifier. IEEE Trans Circuits Syst 1977;24. 